INSTITUT NATIONAL DE RECHERCHE EN INFORMATIQUE ET EN AUTOMATIQUE

\title{
Fair Network Resource Allocation and Link Pricing: A Numerical Study
}

Mustapha Bouhtou, Madiagne Diallo, and Laura Wynter

$\mathbf{N}^{\circ} 4680$

December, 2002

THÈME 4 



\title{
Fair Network Resource Allocation and Link Pricing: A Numerical Study
}

\author{
Mustapha Bouhtou*, Madiagne Diallo ${ }^{\dagger}$, and Laura Wynter ${ }^{\ddagger}$ \\ Thème 4 - Simulation et optimisation \\ de systèmes complexes \\ Projets METALAU
}

Rapport de recherche $\mathrm{n}^{\circ} 4680$ - December, $2002-21$ pages

\begin{abstract}
In this paper, we present an analysis of a restricted bi-level optimization model proposed by [LP98] applied to telecommunication network pricing. This framework provides a generalization of telecommunication resource allocation/shadow price-based schemes such as those of [Kel97] and [LL99], and permits the optimization of link prices with an aim towards revenue maximization. The analysis of the model is based on numerical experiments that we conducted for a variety of networks with varying parameters. We show that in practice the goal of maximizing operator revenue is not always attainable. We present some such cases, as well as valid alternative objectives for these instances.
\end{abstract}

Key-words: Internet Pricing, Network Equilibrium, Proportional Fairness, Bilevel Program

\footnotetext{
* France Télécom R\&D, DAC/OAT,39-40, rue du Général Leclerc, 92131, Issy-Les-Moulineaux, France, Mustapha.Bouhtou@rd.francetelecom.com

$\dagger$ Laboratoire PRISM, Université de Versailles, 45 Av. des Etats-Unis, 78035 Versailles, France, Madiagne.Diallo@prism.uvsq.fr

$\ddagger$ INRIA, Rocquencourt and PRISM, Université de Versailles, Laura.Wynter@inria.fr
} 


\section{L'allocation "équitable" de ressources de télécommunication et la tarification optimale : résultats numériques}

Résumé : Nous présentons un modèle de tarification optimale basé sur une variante simplifié des problèmes bi-niveaux. Ce modèle généralise ceux de [Kel97] and [LL99], en permettant non seulement la définition d'une allocation "équitable" de ressources mais, en parallèle, l'optimisation du revenue du provideur de services de télécommunication. Cependant, contrairement aux modèles "biniveaux" qui sont très ardus à résoudre, celui-ci a l'avantage d'être resolvable par la programmation linéaire.

Mots-clés : Tarification de l'Internet, Equilibres, Optimisation Bi-Niveaux 


\section{Introduction}

The evolution of the telecommunications sector has been characterized by the rapid increase in flow levels as well as the increase in applications making use of telephone and Internet media for transmission. Until recently, the provision of telecommunications services was limited to the national telephone companies. Today, following the de-regulation of the telecommunications markets in most countries, the user is faced with a wide range of choices. A simple connection can involve more than two operators. Because of this multiplicity, it is becoming more important to each operator to examine the pricing of its services. Furthermore, the fact that users have many options available has motivated researchers to consider the user's desires simultaneously with those of the telecom operator in order to optimize the operator's objectives.

Traditionally, models of telecom pricing set prices independently of consumer behavior [AS97], [FO98], and, [FDL00]. A number of recent methods, however, take into account the user by considering his or her willingness to pay, or some QoS requirements, through either a non-linear utility function [GK99], [Kel97], [KMT98], a system of price-equations [CSEZ93], [CESZ91], [Gea97], [MW90], or a pre-defined maximum budget [MMV95].

Models that take into account user behavior, in particular, [GK99], [Kel97], [KMT98] and [LL99], through the incorporation of user utility functions, often define link prices as Lagrange multipliers. While the existence of the prices is thus guaranteed under the hypotheses of the models, the uniqueness is not guaranteed, and in fact, is usually not satisfied, as we shall see in this paper. Furthermore, defining link prices in terms of Lagrange multipliers has one very important consequence. Namely, the price on a link is zero until that link is congested. This property may seem at odds with a provider's goal of revenue maximization; indeed, it was derived rather from the concept of fairness, especially proportional fairness allocation [GK99]. We shall show in this paper that the two notions, that of a fair allocation and of revenue maximization, are not necessarily incompatible.

The objective of this work is to apply optimization models and methods to the management of telecommunications networks through pricing. It is of interest for network resource allocation models and methods to take into account the goal of revenue maximization simultaneously with the maximization of the users satisfaction on the resources provided across the network. To the best of our knowledge, these two elements do not appear to have been dealt with jointly in the literature up to now.

In the next section, we recall the frameworks of Proportional Fairness [GK99] Resource Allocation and Pricing, and Toll Optimization [LP98]. In Section 3, we provide the numerical study and analysis. Section 4 concludes the paper.

\section{Fair Resource Allocation and Revenue Optimization Models}

In some cases, we would like for the prices not to be tied to Lagrange multipliers, but rather to be freely optimized so as to obtain the highest possible revenue. This implies the resolution of two models, which are linked by their common variables: on the one hand, the network operator problem includes prices and total resources allocation, and on the other hand, the users' model determines the level of usage taking into account the different links prices with respect to resources availability.

$\mathrm{RR} \mathrm{n}^{\circ} 4680$ 
Mathematically, this combination of two linked models is formalized as a bi-level, or hierarchical, program. It allows the operator to set network prices and service levels which take into account their effect on the user response. The resulting problem is a highly complex non-convex optimization problem, for which real-time solutions cannot be expected, despite recent advances on computational methods.

In other cases, however, it is desirable for link prices to be defined by Lagrangian multipliers. Proportional Fairness Resource Allocation and Pricing [GK99], [Kel97], [KMT98] advocates this point of view. In particular, a proportionally fair resource allocation $h^{*}$ is one that satisfies for all users, $r \in R$, with a willingness to pay $w_{r}$, the inequality

$$
\sum_{r \in R} w_{r} \frac{\left(h_{r}{ }^{*}-h_{r}\right)}{h_{r}{ }^{*}} \geq 0
$$

for all feasible allocations $h_{r}$. This inequality amounts to the optimality condition of the following optimization program:

$$
\max \sum_{r \in R} w_{r} \log h_{r}
$$

such that $h_{r}$ is feasible with respect to user demand, the capacity constraints on the network links are satisfied, and flows are non-negative. Kelly and colleagues then interpreted the Lagrangian multipliers on the link capacity constraints as the added costs that would drive the allocation to proportional fairness, without imposing them explicitly.

Our approach generalizes the method of pricing suggested by the Proportional Fairness model. In particular, we consider a special case of the bilevel or hierarchical program that has a single, globally optimal, set of network prices. Furthermore, the problem is solvable in polynomial time (for real valued flows), as opposed to its more complex, bi-level, counterpart. To do so, we have adopted the framework of Larsson and Patriksson [LP98] defined for applications in the transport field. Next, we recall this toll optimization framework.

The model combines two problems. When applied to our setting, one must first solve optimally a non-linear resource allocation problem (such as the Proportional Fairness allocation model). Then, one uses the optimal solution and the Lagrange multipliers of this first problem to maximize the operator's revenue function over a restricted set of prices.

Let $G(N, A)$ denote a strongly connected network, where $N$ is the set of nodes and $A$ the set of links. Each link $a$ has associated with it a capacity $u_{a} \in R_{+} \cup\{\infty\}, a \in A$. For certain ordered pairs of nodes, $s=(o, q) \in S \subset N \times N$, there is a demand for communication from $o$ to $q$, known as $d_{s}$. The flow associated with the set of all such demands over the network can be expressed as a flow on each link $a$, or on each origin-to-destination route. We shall assume here that several routes serve the same origin-to-destination pair. The set of all routes on the network will be referred to as $R$. This may represent the case of several physical routes that a request may take, or the presence of more than one network operator serving that pair.

Indicator matrices can be used to convert from the flow on links to flow on routes. Let $\Delta$ be the link-route indicator matrix, where $\Delta_{a r}=1$ if link $a$ is present along on route $r \in R$, and 0 otherwise. 
Let $\Gamma$ be the od-route indicator matrix, where $\Gamma_{r s}=1$ if route $r$ serves the od-pair $s \in S$, and 0 otherwise.

Here, we shall suppose in the remainder of this paper that the users on each od pair are homogeneous in their quality of service (QoS) requirements. Adding user differentiation and/or multiple QoS classes within each od-pair is straightforward, but complicates the notation.

The method of [LP98] works as follows. Consider a canonical network optimization problem with capacity constraints:

$$
\min f(x)
$$

subject to

$$
\begin{aligned}
\Gamma y & =d \\
\Delta y & \leq u \\
d, y & \geq 0
\end{aligned}
$$

where the flow on a link, $a$, is $x_{a}=\sum_{r \in R} y_{r} \Delta_{a r}$. Then, the system based on the Karush-Kuhn-Tucker (KKT) optimality conditions for the problem (3)-(6) as expressed in [LP98] is given below:

$$
T(\lambda, \pi)=\left\{\begin{array}{l}
\Delta^{T}\left[\nabla f\left(x^{*}\right)+\lambda\right]-\Gamma \pi \geq 0, \\
{\left[\nabla f\left(x^{*}\right)+\lambda\right]^{T} x^{*}-d^{T} \pi=0,} \\
\lambda^{T}\left(x^{*}-u\right)=0 \\
\lambda \geq 0
\end{array}\right.
$$

where $\pi$ is the Lagrange multiplier vector for constraints (4). Line 2 of Equation set (7) can be obtained by writing the following linear programming formulation of (3)-(6), solving for $x^{*}$ :

$$
\min _{x} \nabla f\left(x^{*}\right)^{T} x
$$

subject to constraints (4)-(6). The function $\nabla f\left(x^{*}\right)$ is the value of the gradient of the function $f$ evaluated at the optimal solution value, $x^{*}$. While this model is clearly not solvable in practice, since the function value $f\left(x^{*}\right)$ at $x^{*}$ is not known, it permits writing a linear programming dual to the original nonlinear problem (3)-(6). Taking the Lagrangian relaxation of the capacity constraints (5) and adding that into the linear programming objective function (8), we obtain

$$
\begin{array}{ll}
\min _{x} & \nabla f\left(x^{*}\right)^{T} x+\lambda^{T}(x-u)= \\
\min _{x} & \nabla f\left(x^{*}\right)^{T} x+\lambda^{T} x .
\end{array}
$$

Then, the linear programming dual of (9) with constraints (4) and (6) is:

$$
\max _{\pi} \quad d^{T} \pi
$$


subject to

$$
\Gamma^{T} \pi \leq \Delta^{T}\left[\nabla f\left(x^{*}\right)+\lambda\right] .
$$

Strong (linear programming) duality gives the term analogous to that of Line 2 of Equation set (7), that is:

$$
\left[\nabla f\left(x^{*}\right)+\lambda\right]^{T} x^{*}-d^{T} \pi=0
$$

For a fixed, optimal, value of $x^{*}$, the System (7) describes a polyhedron in $\lambda$ and $\pi$. Furthermore, it can be observed that the multiplier vector $\lambda$ will not necessarily be unique in network flow problems.

Indeed, the requirement of linear independence of binding constraint gradients is not always satisfied in networks, as the constraint gradients take the form of indicator matrices for the graph, which are not constructed so as to have linear independent rows. When these multipliers are not unique, then, the authors in [LP98] proposed optimizing a secondary objective over the set of those multiplier values. That is, given the System (7), they posed the following secondary optimization problem:

$$
\begin{gathered}
\max _{\lambda} \phi(\lambda) \\
\text { subject to } \\
\lambda \in T \cap P,
\end{gathered}
$$

where in our setting $T$ is the set of link prices, $\lambda$, given by the System (7), and $\phi: \Re^{|A|} \mapsto \Re$ is coercive, and preferably convex. Since the feasible set is polyhedral, when $P$ is bounded, this secondary, or network manager's optimization problem is easy to solve using standard linear or nonlinear programming algorithms (depending on whether $\phi$ is a linear or nonlinear function of $\lambda$ ).

The network manager's objective can take various forms. Larsson and Patriksson [LP98], in the context of transport planning, discussed at length the network objective of trying to achieve a particular flow of (transport users) by adding prices judiciously; this includes trying to adjust link flows from their user-equilibrium values to a system optimum (using marginal cost pricing) or to some other flow (which then changes the prices that are produced by the model), or even allowing prices to be positive or negative, in which case, travel subsidies are considered (negative prices).

The interest in the model of [LP98] stems from the possibility to optimize network revenue through prices, and to do so using a restricted, and therefore simpler, model than the general bilevel program. Furthermore, the model has the advantage of possessing a unique solution, under very mild and natural conditions.

In [LP98], it was claimed that the maximization of operator revenue in the model they propose, will very often be unbounded, leading to infinitely large prices; in other words, they claim that the revenue maximization problem is usually ill-defined for this model. This outcome is not, however, valid when the network is constructed so as to always contain a non-tolled path, for each user, that is, linking every origin-destination pair. In the case of Internet networks, this assumption will always be verified, as explained further below. When the network is constructed as such, link prices cannot be made arbitrarily large as long as the free path has sufficient capacity. A non-trivial solution (that 
is, non-zero revenue) arises when the free paths offer lower quality of service than the paths tolled by the network operator.

However, in order to be of use in practice, it is necessary to explore the extent to which one can optimize revenue over the polyhedral set of prices, in System (7).

\section{Numerical experience}

The purpose of the tests presented in this section was to evaluate the extent to which link prices that are tied to Lagrange multipliers can be optimized toward revenue maximization. We were therefore interested in evaluating whether the non-uniqueness of the prices that is theoretically possible in the context of the network flow problem arises in practice, and whether it can be optimized sufficiently to allow for revenue maximization.

To that end, by solving the fair network resource allocation problem via an augmented Lagrangian algorithm, relaxing the link capacity constraints, an optimal "fair" link flow, $x^{*}$ is obtained, along with an initial multiplier, or price, vector $\lambda$. With this optimal flow $x^{*}$ to the (for example, proportional) fairness problem in hand along with $\lambda$, it is possible to explore the polyhedron of feasible $\lambda\left(x^{*}\right)$, and, in principle, optimize over that set. We are thus interested in to what extent, in practice this secondary, revenue maximizing optimization is feasible.

The results that follow were all obtained using the Network Equilibrium Toolbox, CiudadSim found at

http://www-rocq.inria.fr/scilab/CiudadSim

in Scilab [Lib] to solve the fair resource allocation problems and the linear programming solver of Scilab to solve the linear revenue maximization problems in $\lambda$.

In the remainder of this section, we provide numerical experience with the models of the previous section. The networks were constructed to represent several possible choices for the user. For each user, characterized by an origin-destination pair, there is always a "best effort" choice, that is, a path with generally higher delay (low QoS) but very high capacity relative to demand levels and consequently not subject to pricing by the network operator. The term "best effort" comes from the current allocation strategy of the Internet: usage is free but no quality guarantees are provided; this service is known as "best effort" service since capacity is allocated to a user "as best as possible".

In addition, to the best effort path available to each user, there is at least one path, or provider, offering service with higher QoS, meaning here lower delay; consequently capacity on those links is lower. The delay functions we chose were quadratic. In our setting we are interested in a particular form of (13). In particular the revenue maximization problem

$$
\max _{\lambda \in T \cap P} \phi(\lambda)=\left(x^{*}\right)^{T} \lambda .
$$

Note that, through the set $P$, this may represent a joint maximization of all providers revenues, or the maximization over a subset of link prices, corresponding to one of several possible providers on the network. 
In the first set of examples, the polyhedral set of prices $T$ will be shown to be a singleton; in other words, the vector of shadow price, $\lambda$, is unique, and therefore no further optimization is possible. In the second set of examples, the set of prices $T$ is not a singleton, and, furthermore, is bounded, thereby permitting further optimization by way of a secondary optimization problem. We thus examine the extent to which operator revenue can be maximized while maintaining the fair allocation. In the last set of examples, we show the possibility of $T$ to be unbounded in the direction of revenue maximization. In other words the objective of global revenue maximization over all non "best-effort" links is not well defined. In this case, we show how one can maximize other objectives such as the revenue over restricted sets of links or routes.

\subsection{Network Instances with Unique Prices}

The first example is performed on a telecommunications network with 5 nodes and 7 arcs, provided in Figure 1. The dashed lines indicate the origin and the destination of a user's request along with the respective demand values, while, the numbers above the solid links indicate the respective optimal link flows. The network data is provided in Table 1. There were two users in this example: one requesting 40 units of service from node 1 to node 5 , the other requesting 50 units from node 2 to node 5 .

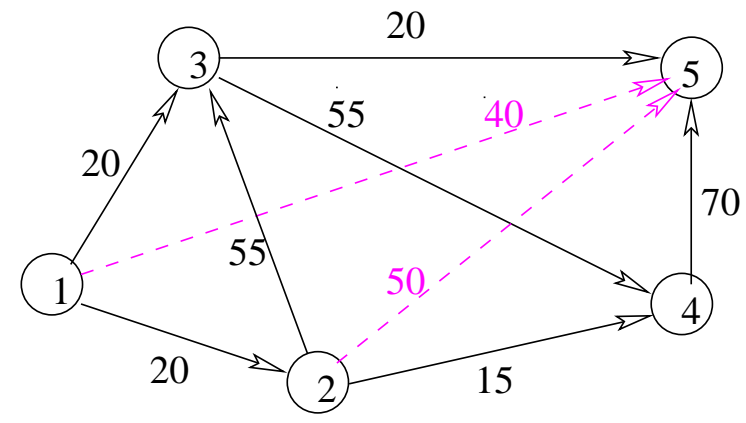

FiguRe 1: Network with Fair Flows

\begin{tabular}{|l|c|c|c|c|c|c|c|}
\hline Link \# & 1 & 2 & 3 & 4 & 5 & 6 & 7 \\
\hline Link Definition & $(1,3)$ & $(1,2)$ & $(2,3)$ & $(2,4)$ & $(3,4)$ & $(3,5)$ & $(4,5)$ \\
\hline Link Capacity & 20 & 100 & 100 & 15 & 100 & 20 & 100 \\
\hline Link Delay & $3 x_{1}{ }^{2}$ & $8 x_{2}+2 x_{2}{ }^{2}$ & $7 x_{3}+2 x_{3}{ }^{2}$ & $8 x_{4}{ }^{2}$ & $9 x_{5}+2 x_{5}{ }^{2}$ & $9 x_{6}{ }^{2}$ & $8 x_{7}+2 x_{7}{ }^{2}$ \\
\hline Fair Link Flow $x^{*}$ & 20 & 20 & 55 & 15 & 55 & 20 & 70 \\
\hline Initial Link Price $\lambda$ & 105 & 0 & 0 & 116 & 0 & 87 & 0 \\
\hline
\end{tabular}

TABLE 1: Network data for Figure 1

Using the augmented Lagrangian method of [LP95a], we obtained the fair resource allocation illustrated in the following network and in Table 1. This solution provides a feasible vector of prices denoted Initial Link Prices $\lambda$ (cf. Table 1). The initial revenue obtained using the initial link prices $\lambda$ is thus

Initial Revenue $=\left(\mathbf{x}^{*}\right)^{\mathbf{T}} \lambda=\mathbf{5 5 8 0}$. 
Given the optimal link flow vector $x^{*}$ (cf. Table 1), we can explicitly express the polyhedron of shadow prices, $T(\lambda, \pi)$, guaranteeing the fair allocation, $x^{*}$, as follows:

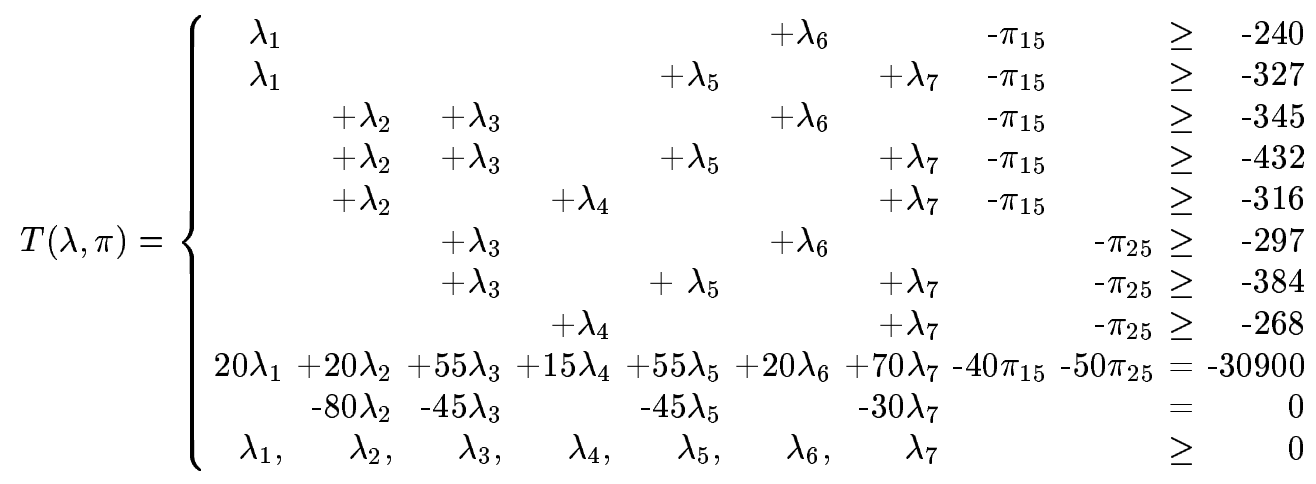

We are interested in checking if the Lagrangian multipliers (shadow prices) of the resource allocation problem are unique or not, that is, whether or not this set $T$ of prices is a singleton. If the Lagrangian multipliers are not unique, the secondary optimization (15) over those prices may produce prices giving higher revenue to the network operator(s).

To do so, we examine the system obtained from the KKT necessary conditions: the Lagrange multipliers are unique if the active inequality and the equality constraints gradients are linearly independent.

From constraints (4) and (5), observe that the equality and inequality constraints gradients are respectively $\Gamma$ and $\Delta$. Thus we can easily identify in $\Delta$ the active constraints, as they correspond, in the arc-route-matrix $\Delta$, to the rows of the saturated arcs. Let us denote the matrix composed of the active and the equality constraints by $I$. From the network data in Table 1, we verify that arcs 1,4 , and 6 are saturated, thus the inequality constraints corresponding to those arcs are active. The matrices $\Delta, \Gamma$ and $I$ are provided below.

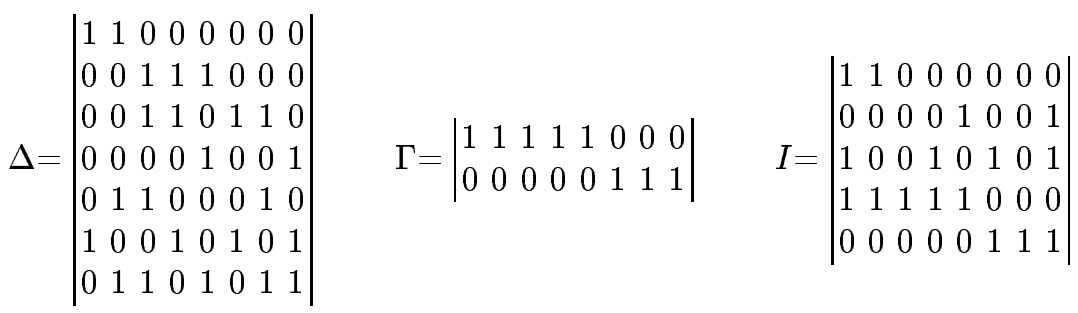

In this example, the rows of matrix $I$ above are indeed linearly independent, since $\operatorname{rank}(I)=5$. In other words, the set of prices $T$ is a singleton with unique element the vector of initial prices in Table 1. Thus, for this network with the given communication demands, no further revenue maximization is possible over this set. Therefore, the maximal revenue is thus equivalent to the initial revenue, which was 5580 .

Figure 2 is the simplest network tested, having only 3 nodes, 3 links, and a single user, from nodes 1 to 3 . The network data is summarized in Table 2 . 
Given the data, and solving for the optimal, fair, resource allocation, in $x^{*}$, only link 2 is saturated. Thus, the matrix of active constraint gradients is

$$
I=\left|\begin{array}{ll}
0 & 1 \\
1 & 1
\end{array}\right|
$$

which is clearly linearly independent. Therefore, the multiplier values are all unique: those on links 1 and 3 are zero, and the only possible value of $\lambda_{2}$ is 672 .

\begin{tabular}{|l|c|c|c|}
\hline Link \# & 1 & 2 & 3 \\
\hline Link Definition & $(1,2)$ & $(1,3)$ & $(2,3)$ \\
\hline Link Capacity & 100 & 20 & 100 \\
\hline Link Delay Data & $4 x_{1}+9 x_{1}{ }^{2}$ & $7 x_{2}$ & $6 x_{3}+3 x_{3}{ }^{3}$ \\
\hline Fair Link Flow $x^{*}$ & 80 & 20 & 80 \\
\hline Initial Link Prices $\lambda$ & 0 & 672 & 0 \\
\hline
\end{tabular}

TABLE 2: Network data for Figure 2

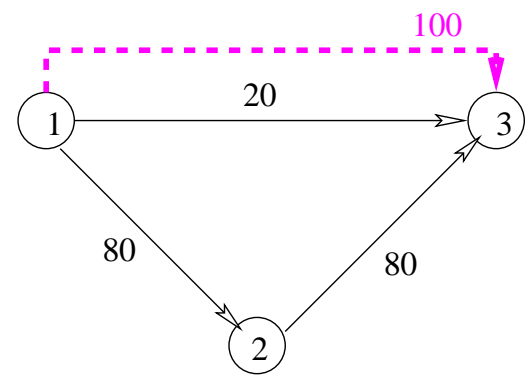

FiguRe 2: Network with Fair Flows

While a number of networks exhibit unique values of the price vector, it should be recalled that this phenomenon is closely related to the network topology. In networks with very few routes, it is likely that the sub-matrix obtained by extracting the rows of the arc-route indicator matrix $\Delta$ which correspond to active capacity constraints, will be linearly independent. This turned out to be the case in some of the examples we constructed. When, however, the number of routes is large, compared to the number of links, and demand is sufficiently high to saturate a large number of links capacities, this linear independence is unlikely to hold. The next examples illustrate the case where link prices are not unique and, furthermore, the set of prices $T$ is bounded.

\subsection{Network Instances with Non Unique Finite Prices}

The present example makes use of the network of Figure 3, and delay data from Table 3. Again, there were two users represented on this network. The first requires 2.5 units from node 1 to 5 ; the second requires 3 units from node 2 to 6 . As before at least one path with high QoS exists for each user as well as a "best effort" choice for each. 


\begin{tabular}{|l|c|c|c|c|c|c|c|c|c|}
\hline Link \# & 1 & 2 & 3 & 4 & 5 & 6 & 7 & 8 & 9 \\
\hline Link Definition & $(1,2)$ & $(1,4)$ & $(2,4)$ & $(2,3)$ & $(2,5)$ & $(3,4)$ & $(3,6)$ & $(4,6)$ & $(6,5)$ \\
\hline Link Capacity & 100 & 1.5 & 100 & 1.8 & 1 & 0.3 & 1.5 & 100 & 100 \\
\hline Link Delay & $5+4 x_{1}$ & $9 x_{2}$ & $7+9 x_{3}$ & $9 x_{4}$ & $4 x_{5}$ & $1 x_{6}$ & $6 x_{7}$ & $4+5 x_{8}$ & $6+2 x_{9}$ \\
\hline Fair Link Flow $x^{*}$ & 1 & 1.5 & 1.2 & 1.8 & 1 & 0.3 & 1.5 & 3 & 1.5 \\
\hline Initial Link Price $\lambda$ & 0 & 0 & 0 & 1.3 & 28.5 & 0 & 10.3 & 0 & 0 \\
\hline
\end{tabular}

TABLE 3: Network Data for Figure 3

Again, using the augmented Lagrangian method, we obtained the fair resource allocation illustrated on the network of Figure 3 and in Table 3. As in Section 3.1, this method provides along with the solution a feasible vector of prices, denoted Initial Prices, $\lambda$. The initial revenue with respect to the fair link flow and the Initial prices, $\lambda$ is

Initial Revenue $=\left(\mathrm{x}^{*}\right)^{\mathbf{T}} \lambda=46.3$.

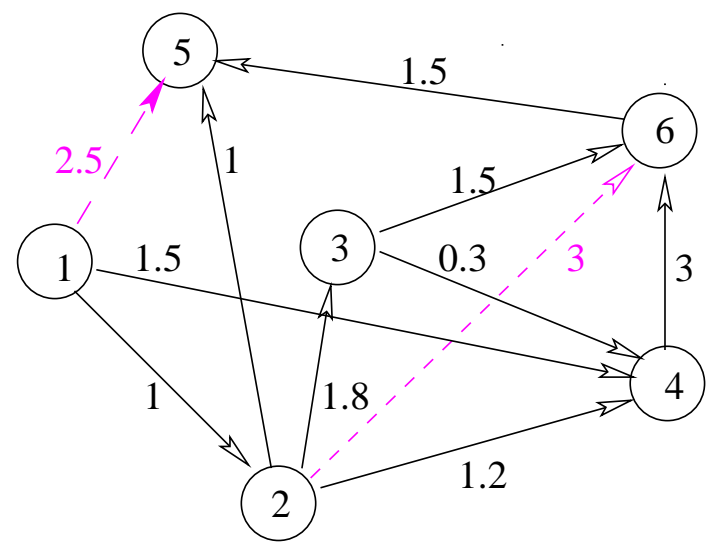

FIgURE 3: Network with Fair Flows

With the fair link flow $x^{*}$ of Table 3 at hand, we write explicitly the set of fair link prices, $T(\lambda, \pi)$, for the present example as follows:

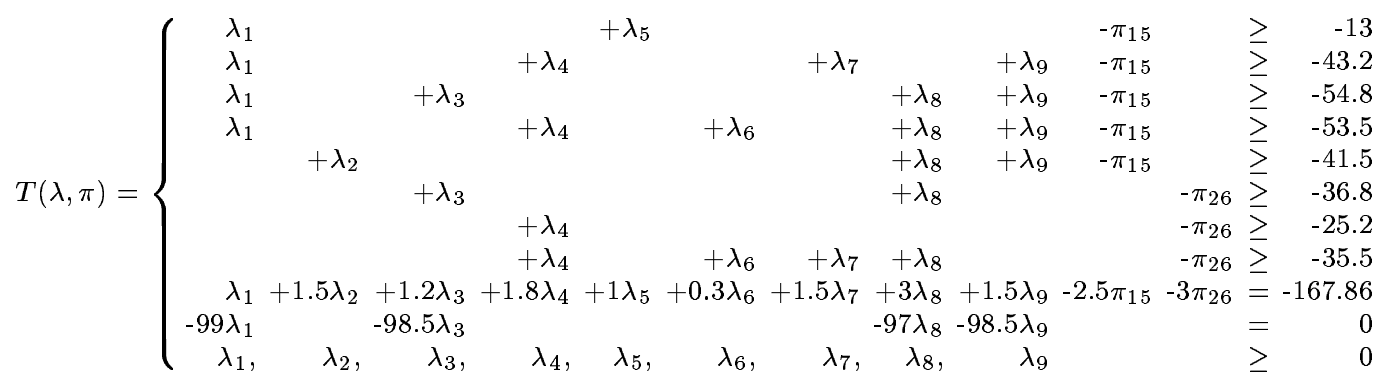


When the multipliers are not unique, the polyhedron of prices $T$ need not be a singleton, and we may be able to maximize the network manager's revenue further than that which was obtained from the initial Lagrange multipliers $\lambda$.

Again, we examine the KKT necessary conditions, denoting by $I$ the set of the active and the equality constraints gradients. It is easy to verify that the capacity constraints relative to arcs 2 , $4,5,6$, and 7 are active since those arcs are saturated. Using the arc-route and od-route matrices, respectively $\Delta$ and $\Gamma$, we construct the set $I$.

For the present example, the matrices $\Delta, \Gamma$ and $I$ are:

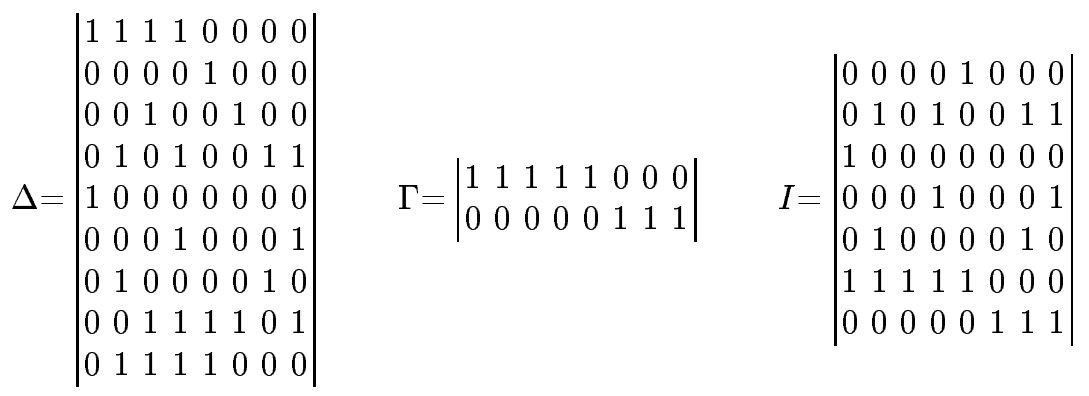

We compute the rank of $I$, which is $6<7$; indeed, summing rows 4 and 5 in $I$ gives row 2 . Thus, the Lagrange multipliers need not be unique and an optimization over the set of prices could be effectively performed.

In this case, we are interested in the extent to which revenue can be maximized by performing the secondary optimization (15) over the set of prices, $T$. In order to do so, we evaluate the size of the polyhedron of feasible prices, $T$, solving the following pair of optimization problems

$$
\min _{\lambda \in T}<e^{i}, \lambda>
$$

and

$$
\max _{\lambda \in T}<e^{i}, \lambda>
$$

We denote $e^{i}$ the $i^{t h}$ coordinate vector, i.e, $i=1, \ldots, 11$, where the 11 variables are the 9 price multipliers, 1 for each arc, and the two demand constraints multipliers, $\pi_{15}$ and $\pi_{26}$. All of the solutions to the above problems were finite; the set $T$ is therefore bounded in all coordinate directions.

The next step is then to solve the revenue maximization problem over the polyhedral (and, in this case, bounded) set of multipliers, $T$. That is, given the fair allocation $x^{*}$, the revenue maximization problem is:

$$
\max _{\lambda \in T} \phi(\lambda)=\left(x^{*}\right)^{T} \lambda
$$

which gives rise to the optimal prices summarized in Table 4 .

Thus, we obtain the maximal revenue

$$
\text { Max Revenue }=\left(\mathbf{x}^{*}\right)^{\mathbf{T}} \lambda^{*}=\mathbf{7 9 . 5 4} .
$$


This value is substantially higher than the initial revenue of 46.3. Recall that the initial link prices were obtained by solving the fair resource allocation problem and taking prices to be the Lagrange multiplier values output by the augmented Lagrangian algorithm. The optimal link prices were obtained by maximizing revenue over the set of Lagrange multipliers. The advantage of solving the secondary linear program is significant in this example.

\begin{tabular}{|l|c|c|c|c|c|c|c|c|c|}
\hline Link \# & 1 & 2 & 3 & 4 & 5 & 6 & 7 & 8 & 9 \\
\hline Optimal Link Prices $\lambda^{*}$ & 0 & 13.3 & 0 & 1.3 & 41.8 & 0 & 10.3 & 0 & 0 \\
\hline
\end{tabular}

TABle 4: Optimal Link Prices for Network of Figure 3

We next provide two other network instances where the polyhedral set of prices is also bounded and not a singleton, thus permitting a secondary optimization to maximize revenue. These networks are represented in Figures 4 and 5. The example of Figure 4 is the largest network studied with 10 nodes and 16 links, where two demands are to be satisfied: one from nodes 5 to 1 requiring 8 units of flow and the other from nodes 3 to 6 with a demand of 13 units. The network data is summarized in the Table 5 .

As in all examples, the link delay functions are quadratic of the form $a x_{i}+b x_{i}{ }^{2}$, where $x_{i}$ is the flow on the link $i, a$ and $b$ are positive. In Table 5. containing the network data, we provide the values of the constants $a$ and $b$, as $(a, b)$.

\begin{tabular}{|c|c|c|c|c|c|c|c|c|c|c|c|c|c|c|c|c|}
\hline Link \# & 1 & 2 & 3 & 4 & 5 & 6 & 7 & 8 & 9 & (10) & 11 & 12 & 13 & 14 & 15 & 16 \\
\hline Link Definition & $(2,5)$ & $(2,7)$ & $(2,8)$ & $(3,2)$ & $(3,10)$ & $(4,2)$ & $(5,4)$ & $(5,6)$ & $(5,9)$ & $(7,1)$ & $(7,5)$ & $(8,6)$ & $(9,1)$ & $(9,4)$ & $(10,4)$ & $(10,8)$ \\
\hline Link Capacity & 100 & 100 & 10 & \begin{tabular}{|l|}
7 \\
\end{tabular} & 100 & 100 & 100 & 100 & 3.7 & 100 & 2 & 10 & 3.7 & 2 & 100 & \begin{tabular}{|l|}
7 \\
\end{tabular} \\
\hline Link Delay Data & $(2,8)$ & $(3,2)$ & $(3,0)$ & $(6,0)$ & $(3,8)$ & $(4,4)$ & $(10,4)$ & $(6,4)$ & $(13,0)$ & $(2,2)$ & $(12,0)$ & $\overline{(2,0)}$ & $(12,0)$ & $(2,0)$ & $(4,5)$ & $(6,0)$ \\
\hline Fair Link Flow $x^{*}$ & 3 & 4.3 & 4 & 7 & 6 & 4.3 & 4.3 & 3 & 3.7 & 4.3 & 0 & 10 & 3.7 & 0 & 0 & 6 \\
\hline Initial Link Prices $\lambda$ & $\overline{0}$ & $\begin{array}{llll}0 & & & \\
\end{array}$ & 3.7 & 4.3 & $\overline{0}$ & 0 & $\begin{array}{c}0 \\
0\end{array}$ & $\overline{0}$ & 0.61 & $\overline{0}$ & $\overline{0}$ & 0.34 & 0.58 & $\overline{0}$ & $\overline{0}$ & $\overline{0}$ \\
\hline
\end{tabular}

TABLE 5: Network Data for Figure 4

In this example, we have from the fair resource allocation and corresponding multiplier values that

$$
\text { Initial Revenue }=52.91 .
$$

The optimal link prices obtained by solving the revenue maximization problem over $\lambda$ is found in Table 6 , and the maximal revenue is

$$
\text { Max Revenue }=100.44 .
$$

Again, a significant increase in network revenue can be obtained by solving the secondary optimization problem over the set of prices, given by the Lagrange multipliers.

\begin{tabular}{|l|l|l|l|l|l|l|l|l|l|l|l|l|l|l|l|l|}
\hline Optimal Link Prices $\lambda^{*}$ & 0 & 0 & 0 & 8 & 0 & 0 & 0 & 0 & 0 & 0 & 0 & 4 & 1.2 & 0 & 0 & 0 \\
\hline
\end{tabular}

TABle 6: Optimal Link Prices for the Network of Figure 4

We turn now to a slight modification on the capacities and delay function constants for the Network of Figure 2. The modified data is given in Table 7. Namely, solving the fair resource 


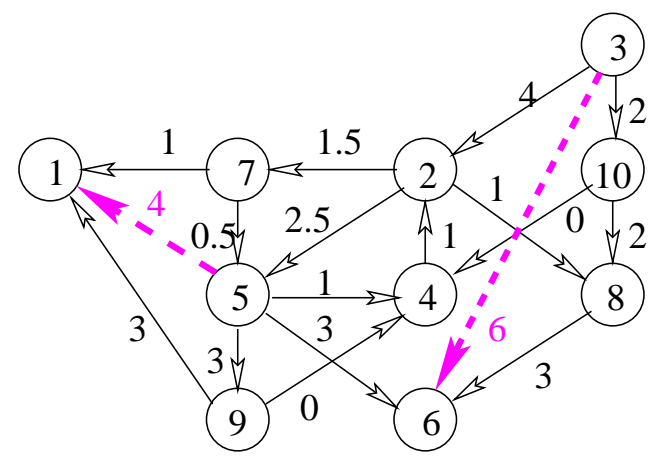

Figure 4: Network with Fair Flows

allocation problem with the modified data, we have now that $\operatorname{arcs} 1$ and 3 are saturated, rendering the matrix of active constraint gradients linearly dependent. As we have seen already, this allows, in principle, for a marge of manoeuvre in setting the multipliers. The initial revenue, read from Table 7 , is equal to $20 * 101.5+20 * 101.5=4060$.

\begin{tabular}{|l|c|c|c|}
\hline Link \# & 1 & 2 & 3 \\
\hline Link Definition & $(1,2)$ & $(1,3)$ & $(2,3)$ \\
\hline Link Capacity & 20 & 100 & 20 \\
\hline Link Delay Data & $(7,0)$ & $(6,3)$ & $(7,0)$ \\
\hline Fair Link Flow $x^{*}$ & 20 & 80 & 20 \\
\hline Initial Link Prices $\lambda$ & 101.5 & 0 & 101.5 \\
\hline
\end{tabular}

TABle 7: Network data for Figure 5

However, optimizing the multiplier values with the aim of revenue maximization, we obtain the optimal link prices below.

\begin{tabular}{|l|c|c|c|}
\hline Link\# & 1 & 2 & 3 \\
\hline Optimal Link Prices $\lambda^{*}$ & 203 & 0 & 0 \\
\hline
\end{tabular}

In other words, while the multiplier values are not unique, and the number of links with non-zero prices can vary, the route prices, in this example, were unique, as is, therefore, the provider's revenue which is equal to 4060 .

\subsection{Unbounded Price Set and Alternative Optimizations}

In this last set of examples, we illustrate the possibility of $T$ to be unbounded in the direction of the profit maximization objective. Note that the present example was obtained by making small changes in the demand values, capacities and delay functions on the data of the Network of Figure 4. Recall that in the example with the original network data of that figure, the set of prices was a singleton. 


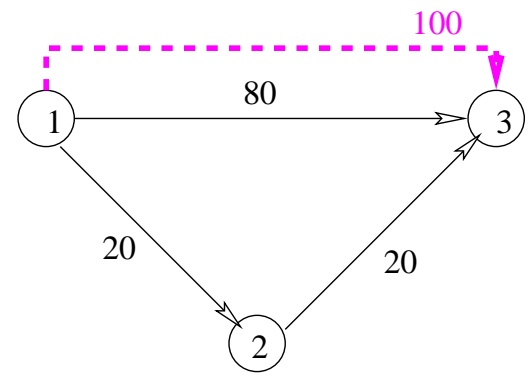

FiguRE 5: Network with Fair (Optimal) Flows

In Table 8, we provide the values of the constants $a$ and $b$ of the delay functions. The network is provided in Figure 6. Two users are represented; the first requests 4 units from node 5 to 1 while the second user requests 6 units from node 3 to 6 . At least one high QoS choice is available to each user, along with "a best effort" option.

\begin{tabular}{|l|c|c|c|c|c|c|c|c|c|c|c|c|c|c|c|c|}
\hline Link \# & 1 & 2 & 3 & 4 & 5 & 6 & 7 & 8 & 9 & $(10)$ & 11 & 12 & 13 & 14 & 15 & 16 \\
\hline Link Definition & $(2,5)$ & $(2,7)$ & $(2,8)$ & $(3,2)$ & $(3,10)$ & $(4,2)$ & $(5,4)$ & $(5,6)$ & $(5,9)$ & $(7,1)$ & $(7,5)$ & $(8,6)$ & $(9,1)$ & $(9,4)$ & $(10,4)$ & $(10,8)$ \\
\hline Link Capacity & 100 & 100 & 10 & 4 & 100 & 100 & 100 & 100 & 10 & 100 & 0.5 & 3 & 3 & 5 & 100 & 2 \\
\hline Link Delay Data & $(12,6)$ & $(10,7)$ & $(16,10)$ & $(0,12)$ & $(8,10)$ & $(6,8)$ & $(4,10)$ & $(4,10)$ & $(0,9)$ & $(10,8)$ & $(0,10)$ & $(0,8)$ & $(0,10)$ & $(0,9)$ & $(11,12)$ & $(0,9)$ \\
\hline Fair Flow $x^{*}$ & 2.5 & 1.5 & 1 & 4 & 2 & 1 & 1 & 3 & 3 & 1 & 0.5 & 3 & 3 & 0 & 0 & 2 \\
\hline Ini. Prices $\lambda$ & 0 & 0 & 0 & 0 & 0 & 0 & 0 & 0 & 0 & 0 & 1.5 & 11 & 9.5 & 0 & 0 & 28 \\
\hline
\end{tabular}

TABLE 8: Network Data for Figure 6

By solving the user problem with the augmented Lagrangian scheme we find the fair link flow $x^{*}$ in Table 8. The Lagrange multipliers obtained jointly with the fair resource allocation $x^{*}$ are the shadow prices $\lambda$ in the same table. For these prices the initial revenue is:

\section{Initial Revenue $=\mathbf{1 1 8 . 2 4}$.}

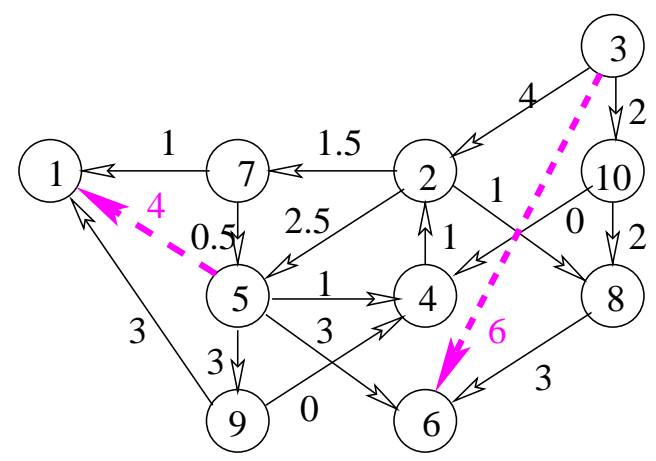

Figure 6: Network with Fair (Optimal) Flows

Using the fair link flow $x^{*}$, we express the polyhedron of shadow prices $T$ as follows:

$$
T(\lambda, \pi)=
$$




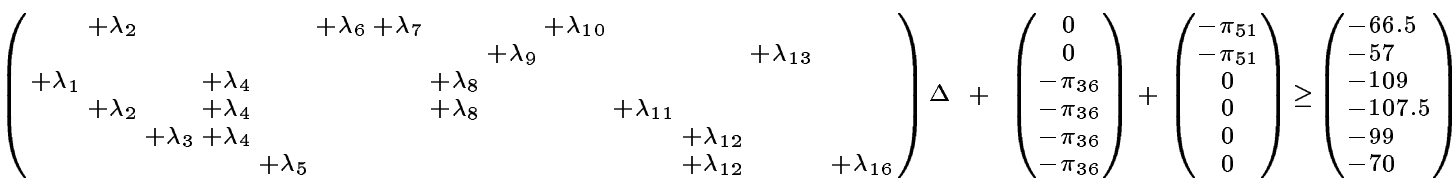

$$
\begin{aligned}
& \left(\begin{array}{rrrrr}
2.5+1.5+1+4 & +2 & +1 & +1 & +3+3+1+0.5+3+3 \\
-97.5-98.5-9 & -98-99-99-97-7-99 & -5-100
\end{array}\right) \Delta+\left(\begin{array}{c}
-4 \pi_{51} \\
0
\end{array}\right)+\left(\begin{array}{c}
-6 \pi_{36} \\
0
\end{array}\right)=\left(\begin{array}{c}
-801.75 \\
0
\end{array}\right) \\
& \lambda=\left(\lambda_{1}, \lambda_{2}, \lambda_{3}, \lambda_{4}, \lambda_{5}, \lambda_{6}, \lambda_{7}, \lambda_{8}, \lambda_{9}, \lambda_{10}, \lambda_{11}, \lambda_{12}, \lambda_{13}, \lambda_{14}, \lambda_{15}, \lambda_{16}\right) \geq 0
\end{aligned}
$$

where, $\Delta$ indicates the arc-route matrix of the network of Figure 6. For the present example, the arc-route $\Delta$, od-route $\Gamma$, and, active and equality gradients constraints $I$ matrices are:

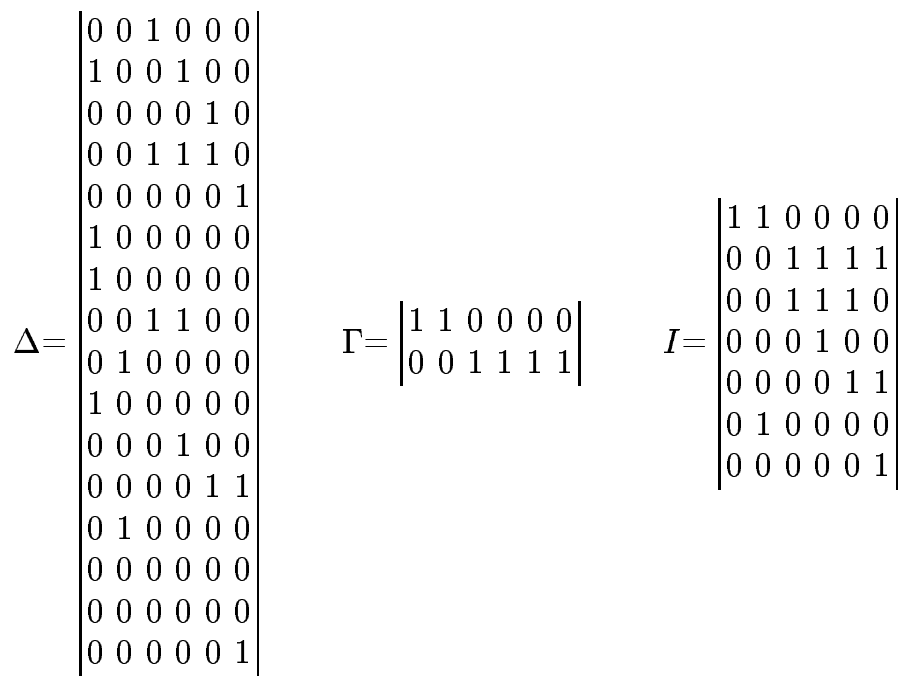

As in the previous example, we applied the same test to determine whether the multipliers are unique or not and to provide an idea of the size of the polyhedron $T$ (summing up rows 3 and 7 gives rise row 2 , thus $I$ is $\mathrm{LD}$ ). In the present example, it happens that at least one of the problems

$$
\min _{\lambda}\left\langle e^{i}, \lambda>,\right.
$$

and

$$
\max _{\lambda}<e^{i}, \lambda>,
$$

is unbounded; namely for $i=4,9,16$, where $\lambda \in T$ and $e^{i}$ is the $i^{t h}$ coordinate vector, i.e, $i=1, \ldots$, 18. The 18 variables are the 16 price multipliers, 1 for each arc, and the two demand constraints multipliers, $\pi_{51}$ and $\pi_{36}$. Thus we see that it is not possible to consider global profit maximization as secondary optimization problem. However, consider selecting a restricted set of arcs such as:

$$
S_{1}=\{11,12,13,14\} .
$$

Then the secondary optimization (15) will attempt to maximize revenue over this smaller set of links. This is of interest if, for example, those links are owned by one operator and a remaining links 
owned by other providers. Alternatively, they may represent a subset of links of one operator which are critical to a particular subnetwork under study. In this case, we solve:

$$
\max _{\lambda \in T} \phi(\lambda)=\sum_{i \in S_{1}} x_{i}^{*} \lambda_{i}
$$

We observe that the optimal solution of $\phi(\lambda)$ is finite and equal to the initial values of the $\lambda_{i}, i \in S_{1}$.

Similarly, it is often of interest to consider a particular set of routes. Consider, for instance the set $R$ of routes $r_{j}$ where

$$
R=\left\{r_{1}, r_{2}, r_{3}\right\} .
$$

and $r_{1}=\{3,12\}, r_{2}=\{11,7,6,3\}, r_{3}=\{14,6\}$. Letting $S_{2}$ denote the links included in route set $R$, we solve, in this case,

$$
\max _{\lambda \in T} \phi(\lambda)=\sum_{i \in S_{2}} x_{i}^{*} \lambda_{i}
$$

Again, we obtain a finite value for the maximal revenue, but again, this value is unique for the selected routes, and is equal to 33.75 .

We next provide another example with an unbounded link price polyhedra. It consists of a network ( Figure 7) with 7 nodes and 13 links with three user demands. Demands are from nodes 2 to 6,4 to 2 and 5 to 4 with respective quantities 40,5 , and 5 . The network data, the resulting fair link flows and the initial link prices obtained with the augmented Lagrangian routine are summarized in Table 9. In this example, although the polyhedral set is unbounded, it is possible to maximize revenue over subsets of arcs or routes, contrary to the above instance in which prices on subsets of links were unique.

\begin{tabular}{|l|c|c|c|c|c|c|c|c|c|c|c|c|}
\hline Link \# & 1 & 2 & 3 & 4 & 5 & 6 & 7 & 8 & 9 & 11 & 12 & 13 \\
\hline Link Definition & $(1,6)$ & $(1,5)$ & $(2,1)$ & $(3,2)$ & $(3,5)$ & $(3,7)$ & $(4,5)$ & $(5,6)$ & $(5,7)$ & $(5,2)$ & $(6,3)$ & $(7,4)$ \\
\hline Link Capacity & 100 & 1 & 100 & 8 & 2 & 100 & 100 & 1 & 2 & 100 & 4 & 100 \\
\hline Link Delay Data & $(4,4)$ & $(6,0)$ & $(7,5)$ & $(3,0)$ & $(6,0)$ & $(9,5)$ & $(8,6)$ & $(6,0)$ & $(6,0)$ & $(8,5)$ & $(7,0)$ & $(6,7)$ \\
\hline Fair Link Flow $x^{*}$ & 39 & 1 & 40 & 0 & 2 & 3 & 5 & 1 & 2 & 5 & 0 & 5 \\
\hline Initial Link Price $\lambda$ & 0 & 74 & 0 & 0 & 4 & 0 & 0 & 74 & 4 & 0 & 0 & 0 \\
\hline
\end{tabular}

TABLE 9: Network Data for Figure 7

In this instance, the initial revenue, as obtained through the initial Lagrange multipliers, is

$$
\text { Initial Revenue }=164 .
$$

Applying the KKT conditions shows that the multipliers are not unique, and, furthermore, solving the pair of optimization problems (16)-(17) shows that $T$ is unbounded in the direction of the objective $\left(x^{*}\right)^{T} \lambda$.

Contrary to the previous example of Figure 6, however, here, we may choose a restricted set of arcs, such as

$$
S_{1}=\{2,4,7,9\} \text {, }
$$

and optimize the revenue over this set. From Table 9, we compute the initial revenue:

Initial Revenue over $S_{1}=82$. 
Solving the following revenue maximization problem over $S_{1}$, we then obtain:

$$
\max _{\lambda \in T} \phi(\lambda)=\sum_{i \in S_{1}} x_{i}^{*} \lambda_{i}
$$

which gives

$$
\text { Max Revenue }=904,
$$

where $\lambda_{i \in S_{1}}^{*}=(148,8,148,8)$.

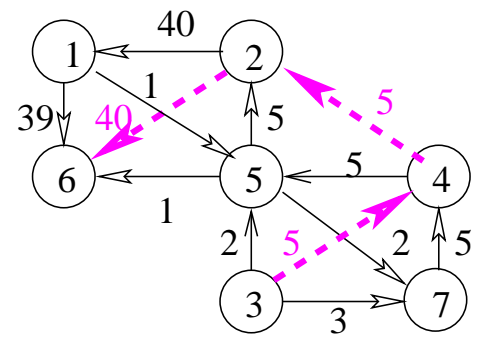

Figure 7: Network with Fair Flows

\section{Perspectives and related results from the literature}

This paper presented the application of a special case of the bilevel programming problem for the solution of a set of fair prices on a telecommunication network, where a network manager optimizes a revenue maximization objective over a particular set. The set is determined by the optimality conditions for a multi-commodity flow problem over the network, subject to capacity constraints. A restriction on the prices states formally that the link prices must be Lagrange multipliers for the capacity constraints, and will therefore be strictly positive only when capacity is reached. This approach generalizes some popular approaches to pricing through fairness, and other Lagrangian schemes for solving telecommunications network flow problems. It draws a parallel between a large body of telecommunications network modeling and the more general bi-level mathematical programming model. A natural next stage of this work would involve developing a characterization of the telecommunication networks that exhibit sufficiently large Lagrange multiplier sets so as to permit considerable revenue maximization. This work also leads naturally into a study of the more general bi-level programming model, by freeing the prices of the complementarity constraints that define them to be Lagrange multipliers.

The numerical exercises have shown that at least, on the quadratic cost fair resource allocation problem, the method of optimizing a secondary objective over the set of Lagrange multipliers, or shadow prices, is worthwhile. Indeed, we have seen that in most instances, revenue maximization is possible for some subnetwork, if not for the entire network, and for some network data. Furthermore, the complexity of the secondary optimization is lower than that of the fair resource allocation problem as it is a linear program, rather than a nonlinear program. An interesting topic for future research would be to test whether the solution to this two-step procedure may come quite close to the true bi-level optimization solution. In other words, the solution to the system described here could be 
used as a good approximation to the true optimal pricing problem in which prices are not restricted to be Lagrange multipliers.

\section{References}

[AS97] L. Anania and R. J. Solomon. Flat: The minimalist price. In L. W. McKnight and J. P. Bailey, editors, Internet Economics, pages 91-118, Cambridge, Massachusetts, 1997. MIT Press.

[CESZ91] Ron Cocchi, Deborah Estrin, Scott Shenker, and Lixia Zhang. A study of priority pricing in multiple service class networks. In SIGCOMM, pages 123-130, 1991.

[CSEZ93] Ron Cocchi, Scott Shenker, Deborah Estrin, and Lixia Zhang. Pricing in computer networks: motivation, formulation, and example. IEEE/ACM Transactions on Networking, 1(6):614-627, 1993.

[DaS00] Luiz A. DaSilva. Pricing for 'qos'-enabled networks: A survey. IEEE Communications Surveys and Tutorials, 3(2), Second Quarter 2000.

[FDL00] M. Falkner, M. Devetsikiotis, and I. Lambadaris. An overwiew of pricing concepts for broadband ip networks. IEEE Communications Surveys and Tutorials, 3(2), Second Quarter 2000. available from URL: http://www.comsoc.org/pubs/surveys/.

[FO98] P. C. Fishburn and A. M. Odlyzko. Dynamic behavior of differential pricing and quality of service options for the internet. In Proc. First Intern. Conf. on Information and Computation Economies (ICE-98), pages 128-139, ACM Press, 1998. Extended version in Decision Support Systems, 28 (2000), pp. 123-136, available from URL http://www.research.att.com/ amo.

[Gea97] A. Gupta et al. Priority pricing of integrated services networks. In L. W. McKnight and J. P. Bailey, editors, Internet Economics, pages 323-352, Cambridge, Massachusetts, 1997. MIT Press.

[GK99] R. J. Gibbens and F. P. Kelly. Resource pricing and the evolution of congestion control. Automatica, 35:1969-1985, June 1999. available from URL http://www.statslab.cam.ac.uk/ frank/evol.html.

[Kel97] F. P. Kelly. Charging and rate control for elastic traffic. European Transactions on Telecommunications, 8:33-37, January 1997. available from URL http://www.statslab.cam.ac.uk/ frank/elastic.html.

[KLO97] Yannis A. Korilis, Aurel A. Lazar, and Ariel Orda. Achieving network optima using Stackelberg routing strategies. IEEE/ACM Transactions on Networking, 5(1):161-173, 1997.

[KMT98] F. P. Kelly, A. K. Maulloo, and D. K. H. Tan. Rate control for communication networks: Shadow prices, proportional fairness, and stability. Journal of the Operational Research Society, 49:237-252, 1998. available from URL http://www.statslab.cam.ac.uk/ frank/rate.html. 
[KVA98] Yannis A. Korilis, Theodora A. Varvarigou, and Sudhir R. Ahuja. Incentive-compatible pricing strategies in noncooperative networks. In INFOCOM (2), pages 439-446, 1998.

[Lib] Scilab Scientific Computing Library. www-rocq.inria.fr/scilab/.

[LL99] S. H. Low and D. E. Lapsley. Optimization flow control, 1: Basic algorithm and convergence. IEEE/ACM Transactions on Networking, 7(6):861-875, December 1999.

[LP92] T. Larsson and M. Patriksson. Simplicial decomposition with disaggregated representation for the traffic assignment problem. Transportation Science, 26:4-17, 1992. available from the URL http://www.math.chalmers.se/ mipat/traffic.html.

[LP94a] T. Larsson and M. Patriksson. An augmented lagrangean scheme for capacitated traffic assignment problems. In N. Bhouri F. Boillot and F. Leurent, editors, Proceedings of the 2nd Meeting of the EURO Working Group on Urban Traffic and Transportation,, pages 163-199, Paris, France, September 15-17 1994. Actes INRETS no. 38, INRETS, Arcueil, Franc. available from the URL http://www.math.chalmers.se/ mipat/traffic.html.

[LP94b] T. Larsson and M. Patriksson. On side constrained models of traffic equilibria. In F. Giannessi and A. Maugeri, editors, Proceedings of the International School of Mathematics "G. Stampacchia" 19th Course on Variational Inequalities and Network Equilibrium Problems, pages 169-178, Erice, Italy, June 19-25 1994. Plenum Press, New York, NY. available from the URL http://www.math.chalmers.se/ mipat/traffic.htmL.

[LP95a] T. Larsson and M. Patriksson. An augmented lagrangean dual algorithm for link capacity side constrained traffic assignment problems. Transportation Research, 29B:433-455, 1995. available from the URL http://www.math.chalmers.se/ mipat/traffic.html.

[LP95b] T. Larsson and M. Patriksson. Equilibrium characterizations of solutions to side constrained asymmetric traffic assignment models. In J. Barcelo, editor, of the 3rd Meeting of the EURO Working Group on Transportation, pages 143-164, Universitat Politecnica de Catalunya, Barcelona, Spain, September 27-29 1995. Institut Catala d'Estudis del Transport. available from the URL http://www.math.chalmers.se/ mipat/traffic.html.

[LP97] T. Larsson and M. Patriksson. Traffic management through link tolls-an approach utilizing side constrained traffic equilibrium models. Rendiconti del Circolo Matematico di Palermo, Serie II 48:147-170, 1997. available from the URL http://www.math.chalmers.se/ mipat/traffic.html.

[LP98] T. Larsson and M. Patriksson. Side constrained traffic equilibrium modelstraffic management through link tolls. In P. Marcotte and S. Nguyen, editors, Chapter 7 in Equilibrium and Advanced Transportation Modelling, pages 125151, Boston, MA, 1998. Kluwer Academic Publishers. available from the URL http://www.math.chalmers.se/ mipat/traffic.html.

[LP99] T. Larsson and M. Patriksson. Side constrained traffic equilibrium models-analysis, computation and applications. Transportation Research, 33B:233-264, 1999. available from the URL http://www.math.chalmers.se/ mipat/traffic.html.

[LPZ97] T. Larsson, M. Patriksson, and W. L. Zhuang. A dual scheme for traffic assignment problems. Optimization, 42:323-358, 1997. available from the URL http://www.math.chalmers.se/ mipat/traffic.html. 
[Mie00] Jan A. Van Mieghem. Price and service discrimination in queuing systems - incentive compatibility of gcu scheduling. Management Science, 46(9):1249-1267, 2000.

[MMV95] Jeffrey K. MacKie-Mason and Hal R. Varian. Pricing congestible network resources. IEEE Journal on Selected Areas in Communications, 13(7):1141-1149, September 1995.

[MW90] H. Mendelson and S. Whang. Optimal incentive-compatible priority pricing for the $m / m / 1$ queue. Operations Research, 38(5):870-883, 1990. 


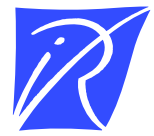

Unité de recherche INRIA Rocquencourt Domaine de Voluceau - Rocquencourt - BP 105 - 78153 Le Chesnay Cedex (France)

Unité de recherche INRIA Futurs : Domaine de Voluceau - Rocquencourt - BP 105 - 78153 Le Chesnay Cedex (France) Unité de recherche INRIA Lorraine : LORIA, Technopôle de Nancy-Brabois - Campus scientifique 615, rue du Jardin Botanique - BP 101 - 54602 Villers-lès-Nancy Cedex (France)

Unité de recherche INRIA Rennes : IRISA, Campus universitaire de Beaulieu - 35042 Rennes Cedex (France)

Unité de recherche INRIA Rhône-Alpes : 655, avenue de l'Europe - 38330 Montbonnot-St-Martin (France)

Unité de recherche INRIA Sophia Antipolis : 2004, route des Lucioles - BP 93 - 06902 Sophia Antipolis Cedex (France) 